\title{
In vitro induction of lymph node cell proliferation by mouse bone marrow dendritic cells following stimulation with different Echinococcus multilocularis antigens
}

\author{
M.C. Margos ${ }^{1}$, D. Grandgirard ${ }^{2}$, S. Leib ${ }^{2}$ and Bruno Gottstein ${ }^{1 *}$ \\ ${ }^{1}$ Institute of Parasitology, Vetsuisse Faculty, University of Bern, \\ Laenggass-Strasse 122, CH-3012 Bern, Switzerland: \\ ${ }^{2}$ Institute for Infectious Diseases, University of Bern, Friedbuehlstrasse 51, \\ CH-3010 Bern, Switzerland
}

(Accepted 2 December 2010; First Published Online 13 January 2011)

\begin{abstract}
The immune response of mice experimentally infected with Echinococcus multilocularis metacestodes becomes impaired so as to allow parasite survival and proliferation. Our study tackled the question on how different classes of E. multilocularis antigens (crude vesicular fluid (VF); purified proteinic rec-14-3-3; purified carbohydrate Em2(G11)) are involved in the maturation process of bone-marrow-derived dendritic cells (BMDCs) and subsequent exposure to lymph node (LN) cells. In our experiments, we used BMDCs cultivated from either naïve (control) or alveolar echinococcosis (AE)-infected C57BL/6 mice. We then tested surface markers (CD80, CD86, MHC class II) and cytokine expression levels (interleukin (IL)-10, IL-12p40 and tumour necrosis factor (TNF)- $\alpha$ ) of non-stimulated BMDCs versus BMDCs stimulated with different Em-antigens or lipopolysaccharide (LPS). While LPS and rec-14-3-3-antigen were able to induce CD80, CD86 and (to a lower extent) MHC class II surface expression, Em2(G11) and, strikingly, also VF-antigen failed to do so. Similarly, LPS and rec-14-3-3 yielded elevated IL-12, TNF- $\alpha$ and IL-10 expression levels, while Em2(G11) and VF-antigen didn't. When naïve BMDCs were loaded with VF-antigen, they induced a strong non-specific proliferation of uncommitted LN cells. For both, BMDCs or LN cells, isolated from AE-infected mice, proliferation was abrogated. The most striking difference, revealed by comparing naïve with AE-BMDCs, was the complete inability of LPS-stimulated AE-BMDCs to activate lymphocytes from any LN cell group. Overall, the presenting activity of BMDCs from AE-infected mice seemed to trigger unresponsiveness in $\mathrm{T}$ cells, especially in the case of VF-antigen stimulation, thus contributing to the suppression of clonal expansion during the chronic phase of AE infection.
\end{abstract}

\section{Introduction}

Dendritic cells (DCs) are highly professional antigenpresenting cells (APC), responsible for the initiation of primary Thelper (Th) cell responses. DCs are specialized

*E-mail: bruno.gottstein@ipa.unibe.ch for detection, uptake and processing of pathogens into small peptides and for the subsequent surfacepresentation on major histocompatibility complex (MHC) molecules. $\mathrm{T}$ cells recognize these antigen fragments by their T-cell receptor (TCR). Additionally to the TCR signal, co-stimulatory molecules, like CD80 (B7-1) and CD86 (B7-2) are necessary for T-cell activation 
(Mellman \& Steinman, 2001), reflecting thus the bond between innate and adapted immunity (Caux et al., 2000). Regulation of immunity in the context of DCs may occur during the initial pathogen-host interaction, or later while establishing a chronic infection (Steinman, 2003; Stettler et al., 2003). With regard to the larval infection with Echinococcus multilocularis, host-parasite interacting parameters focus on the persisting exposure of the host to secretory parasitic products (metabolites), while cell-cell contact appears not to play a central role due to the fact that the living parasite is physically protected by the acellular metacestodic laminated layer (Gottstein et al., 1992). The host-parasite interaction is characterized by an intensive periparasitic inflammatory and granulomatous infiltration leading to an acute necrosis and fibrosis (Gottstein \& Hemphill, 1997) while, in the meantime, an antigen-specific T-cell response is established as well (Bresson-Hadni et al., 1989; Emery et al., 1996). This cellular immune response is crucial for the control of continuous metacestode growth; yet its detailed nature still remains unknown. Principally, impairment of cellular immunity (immune suppression) is followed by an increase in susceptibility to E. multilocularis. This was shown more than 30 years ago in immune suppressed mice (Baron \& Tanner, 1976) and was demonstrated later by using severe combined immunodeficiency (SCID) mice, which were much more susceptible compared to the wild strain and to reconstituted mice (Playford et al., 1992), and in nude mice (Dai et al., 2004). A similar increase of susceptibility, associated with a decrease of delayed type hypersensitivity, was also observed in mice infected with E. multilocularis and treated with an immunosuppressive drug, ciclosporin, which interferes with interleukin-2 (IL-2) production in T cells (Liance et al., 1992).

Conversely, infection in relatively 'resistant' mice showed a stronger cellular immune response (delayedtype hypersensitivity reactions) to parasite antigens (Liance et al., 1990) or specific proliferation of lymphocytes in vitro (Gottstein et al., 1994). That 'resistance' is increased upon stimulation of the cellular immune response was shown with the administration of Bacille Calmette Guérin (BCG) reagent (Rau \& Tanner, 1975), and more recently with Gerbu-adjuvans (Margos \& Gottstein, 2010; Vuitton, 2003). In several past experiments, E. multilocularis was suggested to directly inhibit and/or modulate the immune system either by cell-cell contact or by metabolizing active products. Such mechanisms may occur at the very early stage of antigen presentation to $\mathrm{T}$ cells by DCs and/or macrophages (MØ) (reviewed by Mejri et al., 2010). It was shown that E. multilocularis is modifying the accessory cell function and interferes with the antigen presentation through a parasite-derived macrophage-modifying factor (Dixon, 1997). Furthermore it was shown that immature dendritic cells of human origin did not mature and had a reduced capacity to take up dextran via mannose receptors in the presence of crude, non-fractionated E. multilocularis antigen; however, maturation was inducible by pro-inflammatory cytokines. These mature dendritic cells, pulsed with E. multilocularis antigen, were slightly better inducers of T-cell proliferation than non-pulsed dendritic cells (Jenne et al., 2001). $\mathrm{M} \varnothing$ from alveolar echinococcosis (AE)-infected mice, in their function as antigen-presenting cells (APC), exhibited a reduced ability to present a conventional antigen to specific responder lymph node T cells, when compared to normal MØ (Mejri \& Gottstein, 2006).

Co-stimulatory signals are crucial for T-cell activation and it is well known that failure in the expression of one of the components of the receptor-ligand pairs may severely impair T-cell activation and induce tolerance by a mechanism generally called 'anergy' (Steinman \& Nussenzweig, 2002; Sakaguchi et al., 2008). In the abovementioned experiment, using macrophages from E. multilocularis-infected mice, CD80 and CD86 remained unchanged, whereas CD40 was down-regulated and CD54 (= ICAM-1) slightly up-regulated. Fluorocytometric analyses of peritoneal cells revealed a decrease in the percentage of $\mathrm{CD} 4+$ and $\mathrm{CD} 8+\mathrm{T}$ cells in E. multilocularis-infected mice. Taken together, the obstructed presenting activity of AE-MØ appeared to trigger an unresponsiveness of $\mathrm{T}$ cells, leading to the suppression of their clonal expansion during the chronic phase of AE infection (Mejri \& Gottstein, 2006).

Increasing our knowledge of the interaction of DCs with E. multilocularis antigens may be essential for better understanding the different possible courses of infection/disease, and thus immune effecter and evasion mechanisms. In the present work, we focused our interest on different antigen types of the E. multilocularis metacestode, and their interaction with DCs. The E. multilocularis metacestode is composed of a living, inner, parasitic germinal layer (GL), while the outer, so-called laminated layer (LL) enfolds the whole parasite metacestode to protect it against host reactions (Gottstein et al., 1992; Gottstein \& Hemphill, 1997). One antigen type used in this study was the affinity-purified (Deplazes \& Gottstein, 1991) Em2(G11)-antigen, a major lectin-binding carbohydrate molecule of the LL. Former studies had pointed out (Dai et al., 2001) that Em 2 functions as a T-cell independent (TI) antigen. Em2 doesn't stimulate $\mathrm{T}$ cells to proliferate in vitro, and is only inducing synthesis of low-avidity IgG (Dai et al., 2001). The second, now proteinic antigen model of E. multilocularis we used, was a recombinant 14-3-3 protein (Siles-Lucas et al., 1998; Siles-Lucas \& Gottstein, 2003), the respective native protein being synthesized exclusively within the germinal layer tissue, the 'living' part of the metacestode. Though not secreted, it is nonetheless most likely an important factor in contributing to the control of the basically unlimited metacestode proliferation. Beside its value as a vaccine inducing protection against primary infection (Siles-Lucas et al., 2003), rec-14-3-3 has also been claimed to function as a molecular marker in evaluating the viability and growth activity of the parasite (Matsumoto et al., 2006). Finally, we used a crude vesicular fluid (VF) antigen, generated in vitro by axenical cultivation of E. multilocularis metacestode vesicles (Müller et al., 2007). This VF-antigen was identical to that already used in other immunological studies, including those from Walker et al. (2004) and Dreweck et al. (1999).

\section{Materials and methods}

\section{Echinococcus multilocularis (Em) antigens}

Echinococcus multilocularis vesicle fluid (VF) antigen was obtained by aseptically aspirating fluid from parasite 
vesicles axenically cultivated in vitro according to a procedure described previously (Hemphill \& Gottstein, 1995). The carbohydrate Em2(G11) antigen was purified by affinity chromatography with a solid-phase monoclonal antibody MAbG11-Sepharose matrix (Deplazes \& Gottstein, 1991); E. multilocularis rec-14-3-3 antigen was produced as described previously (Siles-Lucas et al., 1998). The following concentrations were used for in vitro stimulation experiments: VF, $1 \mu \mathrm{g}$ protein/ml; Em2(G11), $2 \mu \mathrm{g}$ carbohydrate/ml; and rec-14-3-3, $1 \mu \mathrm{g}$ protein $/ \mathrm{ml}$.

\section{Infection of C57BL/6 mice with $\mathrm{E}$. multilocularis metacestodes}

Mice were intraperitoneally infected with E. multilocularis clone KF5 metacestode vesicle-suspension as described previously (Dai \& Gottstein, 1999). Three months post-infection, mice were sacrificed by $\mathrm{CO}_{2}$-euthanasia.

\section{BMDC cultures}

Chronically AE-infected, as well as age-matched healthy control, animals were sacrificed for the isolation of bone marrow (BM) progenitors and their subsequent cultivation, as described by Lutz et al. (1999). Culturing took place in Petri dishes (Greiner, 145/20, Sigma Aldrich, Buchs, Switzerland) with feeding of the cells every 3 days with $5 \mathrm{ml}$ RPMI 1640 medium (GIBCO Invitrogen, Basel, Switzerland) and $200 \mathrm{U}$ recombinant human granulocyte-macrophage colony-stimulating factor (rh GM-CSF) (Immunotools, Friesoythe, Germany). On day 9, nonadherent cells were harvested, counted and resuspended to a concentration of $1 \times 10^{6}$ cells $/ \mathrm{ml}$. Cells were plated in 12-well plates (cellstar ${ }^{\circledR}$ Greiner Bio-One, Frickenhausen, Germany) with supplemented medium without GM-CSF for further experiments. The preparation contained 75$80 \%$ CD11c + bone marrow-derived dendritic cells (BMDCs) as determined by flow cytometric analysis.

Sensitization of immature DCs was subsequently carried out with different antigens as follows:

- vesicle fluid (VF) (1 $\mu$ g protein $/ \mathrm{ml})$;

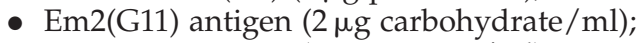

- rec-14-3-3 antigen (1 $\mu$ g protein $/ \mathrm{ml})$;

- lipopolysaccharide (LPS) (100 ng carbohydrate/ml) as a positive control;

- an appropriate volume of medium alone as a negative control.

Stimulation took place overnight (16-18h), cells were then collected, washed with warm supplemented medium and irradiated with $30 \mathrm{~Gy}$.

\section{Flow cytometric analysis}

For phenotypic analyses, stimulated and nonstimulated control BMDCs were fixed with $2 \%$ paraformaldehyde in phosphate-buffered saline (PBS), pH 7.4, and Fc-receptors were blocked by anti-CD16/CD32 MAb. Cells were labelled with fluorochrome-conjugated antibodies against the specific myeloid DC markers CD11c, MHCII CD80, 83 and 86, CD40 (all BD Pharmingen, Allschwil, Switzerland). Naïve control cells were fixed right after BMDC harvest. Data analysis was achieved by using the
CellQuest (BD Biosciences, Allschwil, Switzerland) software. The Excel two-sample $t$-test was used for statistics.

\section{Analysis of cytokine production}

For cytokine measurements, immature DCs were stimulated as outlined above. Supernatants were collected $18 \mathrm{~h}$ after stimulation and samples were then stored at $-20^{\circ} \mathrm{C}$ until further analysis. Cytokine concentrations were measured with the Milliplex ${ }^{\mathrm{TM}}$ mouse cytokine kit (Millipore Corporation, Billerica, Massachusetts, USA) according to the standard protocol provided for culture supernatant. The following cytokines were investigated: IL-10 (Mosmann, 1994), IL-12p40 (Trinchieri, 1993) and tumour necrosis factor- $\alpha$ (TNF- $\alpha$ ) (Carter \& Dutton, 1996). Samples were centrifuged to obtain supernatants without cells. Methodically, samples were investigated undiluted, and fluorescence was measured using the Luminex 200 system (Luminex Corp., Austin, Texas, USA). Samples with undetectable levels of cytokines were attributed a concentration representing the lower limit of quantification, as determined by the manufacturer. Data were analysed employing five-parametric logistic curve fitting using Bioplex manager software 4.01 (BioRad Laboratories Inc., Hercules, California, USA).

Statistical significances $(P$ values $<0.05)$ of differences between cytokine concentration levels of negative controls and experimental assays were determined by two-sample $t$-test, using the NCSS program (Kaysville, Utah, USA).

\section{Cell proliferation assay}

Lymph node (LN) cells (renal, inguinal, lumbar and brachial) from naïve or E. multilocularis AE-infected mice were isolated as described previously (Dai \& Gottstein, 1999). LN cells $\left(1 \times 10^{6} / \mathrm{ml}\right)$ were co-cultivated with BMDCs $\left(1 \times 10^{5} / \mathrm{ml}\right)$ from naïve or AE-infected mice. These BMDCs had previously been cultured in medium alone or stimulated with LPS or different Em antigens for 18h. They were washed thereafter and irradiated (30 Gy), as described in a previous section. After 5 days of co-cultivation, cells were pulsed with $1 \mu \mathrm{Ci}$ / well $\left[{ }^{3} \mathrm{H}\right]$ thymidine. Eighteen hours later, cells were harvested on to glass fibre filters and activities were measured in a TopCount NXT (Packard Instruments) by thymidine incorporation. Statistics were performed using the NCSS program.

\section{Results}

\section{Surface molecule expression}

Naïve or AE-BMDC cultures, kept overnight in enriched medium alone, exposed to LPS or different Em antigens, were subsequently collected and analysed by fluorocytometry. Results are presented in table 1 . Immature DCs displayed phenotypical $\mathrm{MHCII}^{\text {low }}$, CD80 ${ }^{\text {low }}$ and CD86 ${ }^{\text {low }}$. Relevant surface markers known to be up-regulated upon DC maturation revealed no differences in their expression levels between naïve and infected mice after stimulation with LPS. We were interested in seeing whether Em antigens, different in their biochemical composition, do induce maturation of 


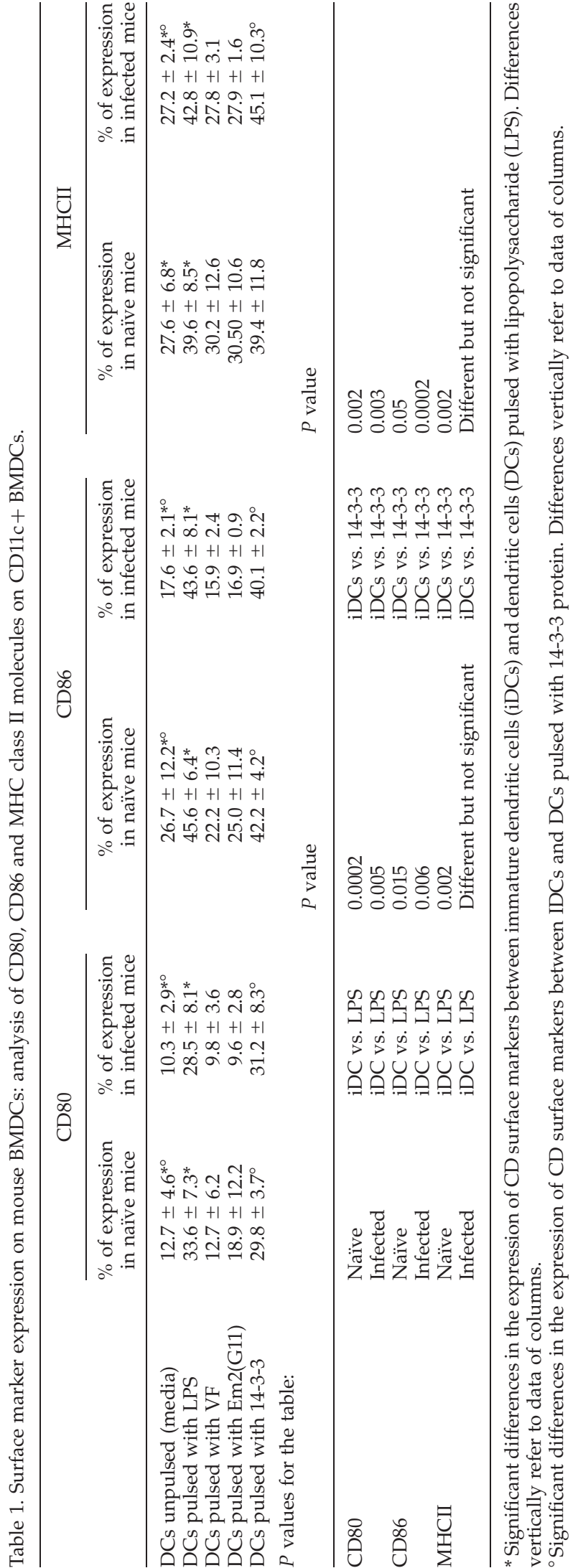

BMDCs, a principally crucial step for antigen presentation capacity of DCs. All DC surface markers showed, as expected, a significant up-regulation after LPS stimulation compared to the presentation on immature DCs. No maturation on DCs was, however, detected after VF and Em2(G11) incubation for any of the markers. Stimulation with rec-14-3-3 displayed a significantly higher CD80 expression on the surface of BMDCs (naïve, $P=0.002$; infected, $P=0.003$ ), comparable to LPS stimulation (naïve, $P=0.0002$; infected, $P=0.005$ ). A similar significant rise was found in CD86, whereas for MHC class II expression the level was raised but not significantly.

\section{Cytokine expression profiles}

DC cultures, identically processed as described in the previous paragraph (overnight incubation with Em antigens or LPS) were used to collect supernatants, which were stored frozen at $-20^{\circ} \mathrm{C}$ until further analysis by the Luminex cytokine system (fig. 1). IL-10 production rose significantly after LPS and after rec-14-3-3 stimulation when compared to non-stimulated BMDCs (fig. 1A). VF and Em2 (G11) had no effect on DCs and their production of this cytokine. Similar results were found for IL-12 (fig. 1B) as well as TNF- $\alpha$ (fig. 1C) production. Non-stimulated, VF and Em2(G11) stimulated DCs did not produce appropriate cytokines above detection limit, while the stimulation with LPS and rec14-3-3 gave rise to significantly elevated levels compared to non-stimulated cells. No significant differences were found in the cytokine production between BMDCs from naïve and AE-infected mice, but IL-12 production after LPS and rec-14-3-3 stimulation appeared slightly (but not significantly) elevated in BMDCs from AE-infected mice.

\section{Lymph node cell proliferation}

The efficiency of BMDCs pulsed with different Em antigens were assessed for their potential to induce LN cell proliferation. Therefore, BMDCs were co-cultured with LN cells from AE-infected or non-infected naïve control mice. LN cells were pooled from five mice respective to each group. BMDCs were phenotypically analysed by flow cytometric analysis (fig. 2). LPS stimulated BMDCs (mature DCs) from naïve mice induced proliferation of LN cells from both AE-infected and noninfected control groups, when compared to immature DCs (fig. 2A). The stimulation of naïve BMDCs with VF showed a significantly $(P=0.005)$ higher proliferative response in non-infected mice than LN cells from AE-infected mice, the latter remained non-responsive. The carbohydrate Em2(G11) yielded no response in naïve mice LN cells, while this antigen gave rise to thymidine incorporation in AE-infected mice, although the difference remained statistically not significant. BMDC stimulation with rec-14-3-3 led to a stimulation profile relatively similar to that of $\mathrm{VF}$, with a high proliferative response in control mice, and a respectively reduced response in AE-infected animals. With regard to the use of BMDCs isolated from AE-infected mice, all experiments with LN cells from non-infected control groups showed baseline reactivity (fig. 2B), even following those 

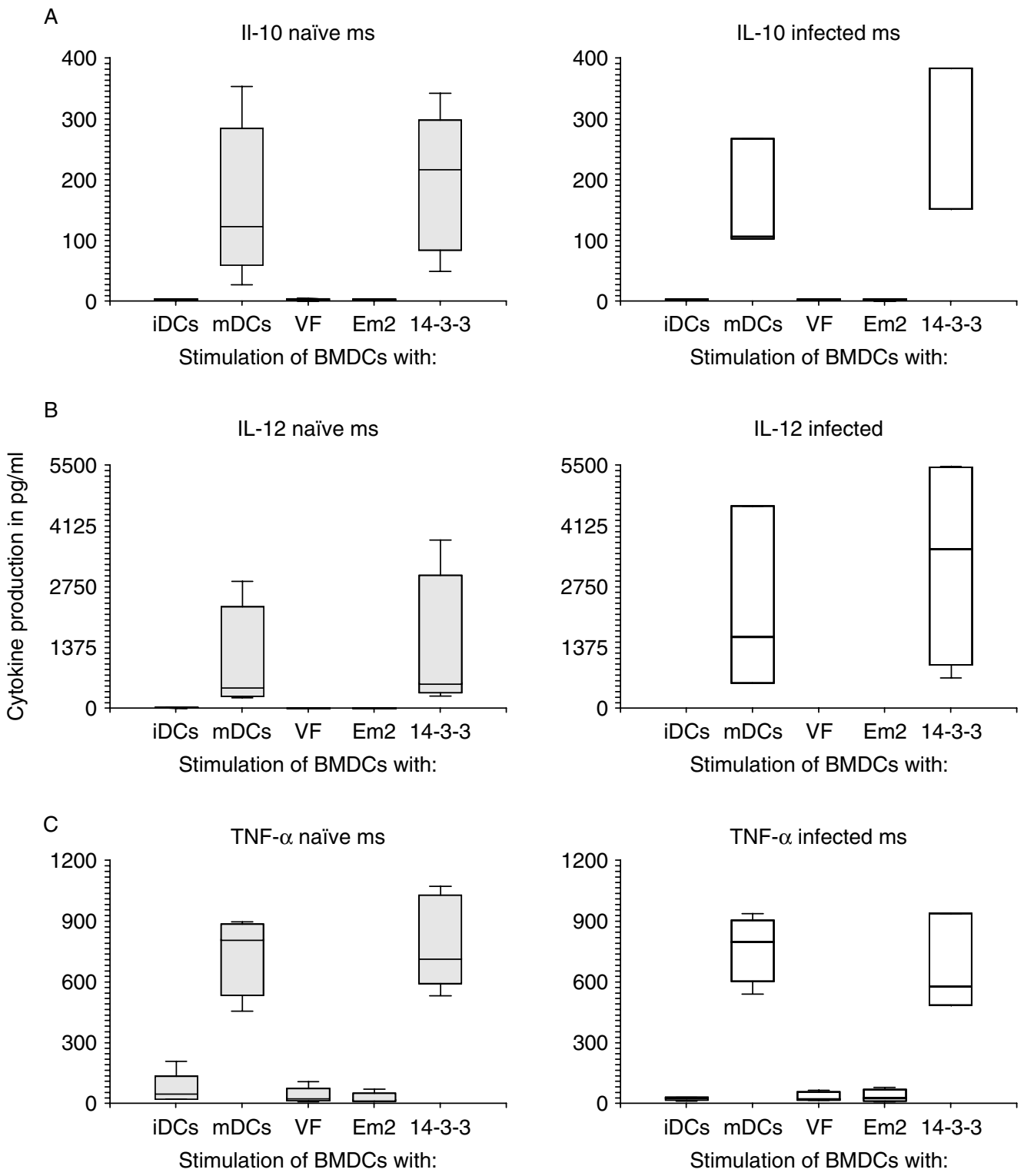

Fig. 1. Cytokine detection by microsphere-based multiplex procedure. Cytokine concentrations in $\mathrm{pg} / \mathrm{ml}$ were measured for interleukin (IL)-10 (A), IL-12p40 (B) and tumour necrosis factor- $\alpha$ (TNF- $\alpha$ ) (C) in supernatants from dendritic cells (DCs) (isolated either from naïve or AE-infected mice) maintained in vitro as follows: untreated (iDCs), stimulated with LPS (mDCs) or with different antigens (VF, Em2, 14-3-3) for 16-18 h.

stimulations that had induced proliferation with naïve mouse BMDCs (LPS, VF and rec-14-3-3). In AE-infected mice, LPS stimulation (mature DCs) failed to induce proliferation, while VF- and rec-14-3-3-stimulation initiated respective proliferation. As for naïve mice, Em2(G11) yielded only a small, statistically not significant response with LN cells from AE-infected mice.

\section{Discussion}

Dendritic cells (DCs) are a unique type of professional antigen-presenting cells involved in linking innate and adaptive immunity. The initiation of Th1 or Th2 immunity is dependent on the involved antigen biology and the influence of cytokines (Sallusto \& Lanzavecchia, 2002). Th1 type immunity onset is a really fast process triggered by a high IL-12 production by DCs (Macatonia et al., 1995; Carter \& Dutton, 1996). While these processes inducing Th1 immunity are well described, the induction of a Th2 type immune response, often found in helminthic infections (Maizels \& Yazdanbakhsh, 2003) still requires enlightenment. As it is not clear whether DCs are really playing a central and direct role in promoting a Th2 response, it sparked our interest in bone marrow 


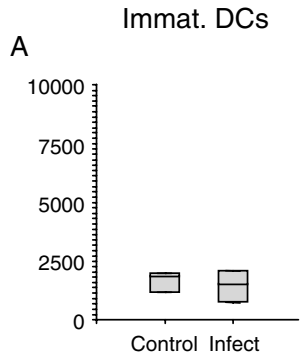

B

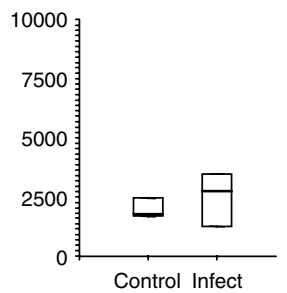

Mature DCs
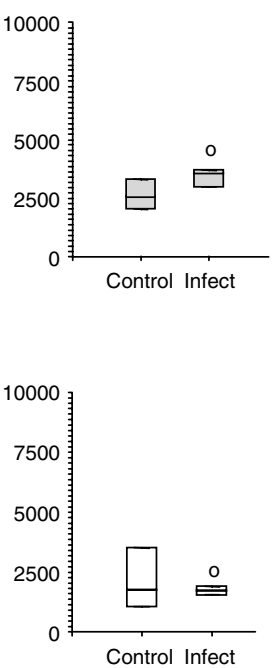

VF stim. DCs
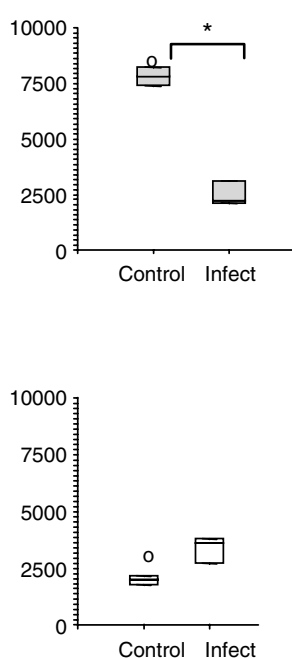

Em2 stim. DCs
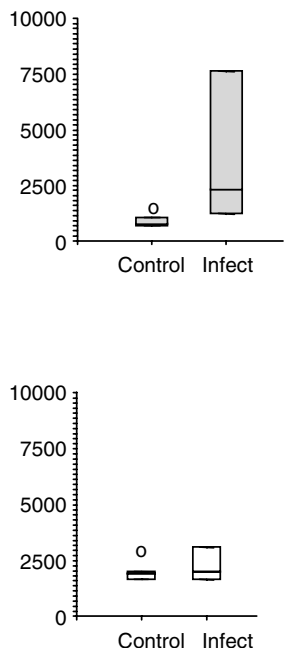

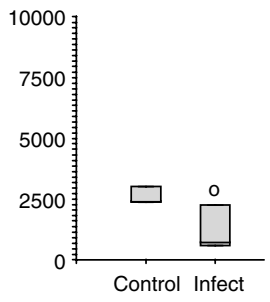

14-3-3 stim. DCs

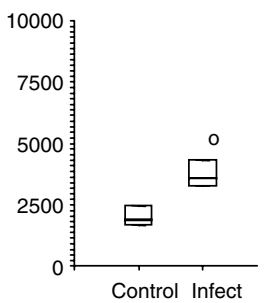

Fig. 2. Assessment of different dendritic cells (DCs) to induce lymph node (LN) cell proliferation. LN cells from naïve control (A) and AE-infected (B) mice, were co-cultivated for 16-18 h with differently stimulated bone-marrow-derived dendritic cells (BMDCs) (immature; mature/LPS; VF, Em2(G11) and rec-14-3-3 stimulation). Significant differences of VF-stimulated DCs from control mice between control and infected LN cells are labelled with *, while significant differences between LN cells from naïve (A) and infected (B) mice are labelled by ${ }^{\circ}$ (vertically oriented association).

dendritic cells (BMDCs) and we were keen to see if different kinds of E. multilocularis antigens may influence BMDCs in their cytokine production, the ability to express surface molecules or in their potential for antigen presentation to lymphocytes and subsequent induction of a proliferative process. In the case of surface markers, the stimulation with Em2(G11) and VF-antigen displayed a phenotype of DC surface markers resembling that of an immature status; CD80 ${ }^{\text {low }}, \mathrm{CD}^{\text {low }} 6^{\text {and }} \mathrm{MHCII}{ }^{\text {low }}$, no maturation was traceable. Former reports (Konecny et al., 1999; MacDonald et al., 2001) had already shown that parasites, and especially helminths, do not seem to be efficient in inducing DC maturation, at least when looking at expression of classical maturation markers, such as CD80, CD86 and MHC class II. Therefore, our findings were not really surprising. Among the different classes of antigens we tested, only the proteinic rec-14-3-3 was able to up-regulate the surface markers. DCs pulsed with the crude VF-antigen failed in this respect. Previous studies (Mejri \& Gottstein, 2006) had already demonstrated a reduced presenting activity of E. multilocularisinfected host MØs, which appeared to trigger an unresponsiveness of $\mathrm{T}$ cells, leading to the suppression of their clonal expansion during the chronic phase of AE infection. Similar mechanisms may now also hold true for DCs. The Em2(G11) antigen is a major carbohydrate localized primarily in the laminated layer of the metacestode (Gottstein et al., 1992). Em2 was shown to be a T-cell-independent antigen, and the corresponding antibody response lacked antibody maturation (Dai et al., 2001). Thus, the failure to induce an appropriate maturation of DCs wasn't really surprising. Further, it also had been shown that immature dendritic cells of human origin are not maturing and have a reduced capacity to up take dextran via mannose receptors in the presence of crude non-fractionated E. multilocularis antigen (Jenne et al., 2001). Another study with a hydatid cyst component antigen of E. granulosus (AgB) had already revealed a modulation of the immune system in the human intermediate host. In these experiments, it seemed that this antigen was impairing the differentiation of monocyte precursors into iDCs, rendering them unable to mature in the presence of LPS; in addition the authors found that DCs that had matured with this antigen exhibited a reduced capacity of responding to inflammatory stimuli, such as LPS (Riganò et al., 2007). Additional studies will be needed to elucidate in more detail the role that parasitic (metacestode) carbohydrates may play in the modulation of antigen-presenting capacities of host DCs and MØ.

A stable ratio of IL-10 and IL-12 is an important feature for the balance of Th1 and Th2 immune responses (Meyaard et al., 1996). In experimental AE, it had been previously demonstrated that an initial Th1-oriented response was gradually replaced by a Th2 response in the later stage of infection, as assessed by cytokine and lymphocyte patterns in vitro (Wei et al., 2004). Thus, we were interested to see whether different metacestode antigens influence BMDCs and respective cytokine production. Experimentally, neither Em2(G11) nor VF stimulation induced any cytokine production by BMDCs, not for naïve nor for AE-infected animals. Conversely, LPS and parasitic rec-14-3-3 stimulations were able to induce measurable cytokine production by BMDCs. We tested IL-12, a first-instance cytokine against pathogens and an important molecule for the optimal generation of CD4 + cells (Trinchieri, 1993); the results after LPS stimulation were as expected, i.e. significantly increased IL-12 levels after the stimulation of both naïve and AE-BMDCs. Rec-14-3-3-stimulated cells produced high 
amounts of this cytokine. When comparing naïve versus AE-infected mice, a slightly higher, but statistically not significant, amount of IL-12 production was observed in AE-mice (LPS, $P=0.05$; rec-14-3-3, $P=0.06$ ). Consequently, only rec-14-3-3 was able to induce IL-12 production in vitro in BMDCs; normally this protein lies hidden in the metacestode tissue, not exposed to the host and its immune system. But artificially exposed to BMDCs, as in our experiment, it seems to induce a rather Th1-oriented cytokine profile not found in vivo. Further investigation may address this by super-immunization of an already infected mouse; this may support a reorientation of the response in the direction of Th1, which may then be of benefit for the host by rendering it more resistant to infection. The failure of Em2 and VF-antigen to induce an appropriate response may resemble that found by MacDonald et al. (2001), who used the soluble egg antigen (SEA) of Schistosoma mansoni to stimulate BMDCs and found that this antigen was not able to induce any up-regulation of surface molecules like CD80, CD86 and MHC class II, nor were any respective cytokines produced, when compared to LPS-stimulated BMDCs. But, nonetheless, SEA was able to support the development of a Th2-oriented response in vitro and in vivo (MacDonald \& Pearce, 2002). IL-10 induction, amongst other reactions, is followed after the release of IL-12 by monocytes or other APCs (Trinchieri, 2003). So, after recognizing an increase of IL-12 in BMDCs of infected mice, we were interested in seeing whether IL-10 levels were also increased, as this cytokine plays the counteracting part to IL-12. In schistosomiasis, this cytokine is known to have an important function in suppressing a needed Th1 response, allowing the parasite's survival in its host by preventing a severe inflammatory response by this change of immunity (MacDonald et al., 2002). In former AE studies, IL-10 production was only assessed in lymphocytes and an increased production was revealed (Bresson-Hadni et al., 1989). In the present study, the IL-10 production of BMDCs from infected mice was only elevated after LPS or rec-14-3-3 stimulation, again, and no differences occurred in IL-10 levels between naïve and AE-infected mice. Yet a negative feedback regulation of the immune response on IL-12 (D'Andrea et al., 1993) may be due to only a small elevation of IL-10, as found in our experiments with regard to LPS and rec-14-3-3 stimulation. IL-10 not only acts at the beginning of an infection, it may also be involved in a later step, suppressing the synthesis of several other inflammatory mediators and promoting a Th2 response (Mosmann, 1994). The VF-antigen used is predominantly an excretory and secretory (E/S) product of the E. multilocularis metacestode. Similarly metabolized molecules may also be found in other extracellular plathelminthic infections. They are mostly antigenic and are supposed to have immune-modulatory properties (Lightowlers \& Rickard, 1988; Harnett \& Harnett, 2008). Their influence may determine the outcome of infection in favour of the parasite. It is thought that the modulation of the immune response is predominating at the chronic phase of AE infection; but finding that BMDCs stimulated with VF-antigen were only able to induce proliferation in naïve LN cells, while LN cells from AE-infected mice showed no reaction, may indicate other or additional immune-modulating activities. One should consider that E/S molecules may also be involved in the early phase of infection in the down-regulation of the host's immune response (Jenkins et al., 2005). In schistosomiasis (PeronaWright et al., 2006), cercarial E/S products are assumed to skew the phenotype of DCs, maybe by release of cytokines upon different routes, promoting therefore a Th2-response in lymphocytes or other immune cells. Antigenic metabolites of E. multilocularis may act similarly. Furthermore, it may be that helminths are acting on DCs and macrophages in such a way as to express different surface immune receptors, as assumed in schistosomiasis and its product $0-2 \mathrm{~h}$ RP (Jenkins et al., 2005). In vitro, VF is showing similar inhibiting reactions to peripheral blood mononuclear cells (PBMCs) as cercarial preparations of Schistosoma mansoni (Jenkins et al., 2005). Summarized, it seems that Em2 and VF are not involved in a normal maturation induction of BMDCs, nevertheless they might be involved in different steps, not yet detected. Toll-like receptors (TLR) are an important parameter in innate immunity, enabling DCs to recognize pathogen-associated molecule patterns (PAMPs), like LPS (Barton \& Medzhitov, 2003). Cytokine secretion by DCs may follow as initiation of a local inflammatory process and cell differentiation in the lymphoid tissue (including antigen processing, up-regulation of co-stimulatory molecules and T-cell activation) (Pasare \& Medzhitov, 2004). The exposure of DCs to LPS always yielded mature DCs, as evidenced by up-regulation of co-stimulatory molecules and induction of lymphocyte proliferation. The laminated layer antigen Em2(G11), in constant close contact with the adjacent host tissue, may therefore be involved in a modulation process as mentioned above. No obvious changes after stimulating BMDCs with this carbohydrate antigen were detectable, either in expression of co-stimulatory molecules, or in the ability to activate LN cells. Maybe Em2(G11) is disguised from conventional presentation; former studies have already documented that the host is producing antigenspecific IgM and subsequently switching to IgG without the help of CD4+ T cells (Dai et al., 2001). As mentioned above, upon AE infection, the murine host starts with a Th1 response which is induced by IL-12 and other factors (Emery et al., 1996; Godot et al., 1997; Jenne et al., 1998; Liance et al., 1998; Godot et al., 2003). This response harbours the potential of a rather 'protective' or parasitegrowth-inhibitory effect. In progressing murine $\mathrm{AE}$, however, this response is gradually substituted by a Th2-oriented response, which now is in favour of the parasite's survival and subsequent growth. In a last step of the experiments, we investigated the proliferative behaviour of LN cells (isolated from naïve or AE-infected mice) and their response to BMDCs, which were previously sensitized with different antigens. For naïve BMDCs, the striking observation was that LN cells from uncommitted naïve (control) mice were responding to VF-antigen presentation, in contrast to LN cells from AEinfected mice, and in contrast also to the same experiments carried out with LN cells from AE-infected mice. This raises the assumption that putative immune suppression or evasion mechanisms, triggered by the AE-infection, may cause non-recognition of VF-antigen in the host at several levels, including that of DC-processing 
in AE-infected mice and antigen-recognition by LN cells from AE-infected mice. VF-antigen on its own, however, is able to induce a non-specific response, as shown in the naïve-naïve animal experiments, where Em2(G11)sensitized BMDCs displayed no effect in their presenting ability to either naïve or AE-infected LN cells. Yet this was not really surprising, as Dai et al. (2001) had already observed a similar lack in conventional lymphocyte proliferation. Results obtained with rec-14-3-3 were roughly as expected, although slightly biased by a background proliferation that was probably due to minor bacterial contaminations. Rec-14-3-3-loaded BMDCs exhibited no significant effect on naïve LN cells, whereas stimulation was observed in LN cells from AEinfected mice. The effect remained, however, relatively low. The most striking difference, revealed by comparing naïve with AE-BMDCs, was the complete inability of LPS-stimulated AE-BMDCs to activate lymphocytes from any LN cell groups, although an appropriate expression of co-stimulatory molecules had been demonstrated on the surface of both naïve and AE-BMDCs. The detected inability of antigen presentation must have its origin in other molecular mechanisms.

The interesting fact that BMDCs from infected mice showed no, or only weak, responses to any of the stimulations, raises the question of how far this parasite is triggering a down-regulation of the immune response in its host at a very initial and basic level. Is this parasite even able to act on cells in the bone marrow? Varol et al. (2007) suggested that the old scheme of myeloid differentiation as a 'one way' intermediate from the bone marrow to the periphery has to be revised, as blood monocytes can efficiently home back to the bone marrow. Another group (Becker et al., 2005) found that CD8+ memory T cells can also reside in the BM and thus can respond faster to antigens than cells residing in other tissues. Combined, this may suggest a pathway allowing DCs to collect pathogens at peripheral sites and subsequently transport them to the BM where DCs could induce a rapid proliferation of resident CD8+ memory cells. The effect (already shown for Listeria monocytogenes) is a so-called Trojan horse mechanism, and maybe E. multilocularis is making use of a similar mechanism of disguise at onset of infection.

So far, a complete picture of how the metacestode escapes host immunity has not yet been elucidated, but step-by-step we are putting together pieces of the puzzle toward understanding how this parasite survives in its host.

\section{Acknowledgements}

Andrew Hemphill (Institute of Parasitology, University of Bern) is greatly acknowledged for his helpful comments and discussion. This work was supported by the Swiss National Science Foundation (grant no. 3100A0-111780).

\section{References}

Baron, R.W. \& Tanner, C.E. (1976) The effect of immunosuppression on secondary Echinococcus multilocularis infections in mice. International Journal of Parasitology 6, 37-42.

Barton, G.M. \& Medzhitov, R. (2003) Toll-like receptor signaling pathways. Science 300, 1524-1525.

Becker, T.C., Coley, S.M., Wherry, E.J. \& Ahmed, R. (2005) Bone marrow is a preferred site for homeostatic proliferation of memory CD8 T cells. Journal of Immunology 174, 1269-1273.

Bresson-Hadni, S., Vuitton, D.A., Lenys, D., Liance, M., Racadot, E. \& Miguet, J.P. (1989) Cellular immune response in Echinococcus multilocularis infection in humans. I. Lymphocyte reactivity to Echinococcus antigens in patients with alveolar echinococcosis. Clinical and Experimental Immunology 78, 61-66.

Carter, L.L. \& Dutton, R.W. (1996) Type 1 and type 2: a fundamental dichotomy for all T-cell subsets. Current Opinion in Immunology 8, 336-342.

Caux, C., Ait-Yahia, S., Chemin, K., de Bouteiller, O., Dieu-Nosjean, M.C., Homey, B., Massacrier, C., Vanbervliet, B., Zlotnik, A. \& Vicari, A. (2000) Dendritic cell biology and regulation of dendritic cell trafficking by chemokines. Springer Seminar in Immunopathology 22, 345-369.

D'Andrea, A., Aste-Amezaga, M., Valiante, N.M., Ma, X., Kubin, M. \& Trinchieri, G. (1993) Interleukin 10 (IL-10) inhibits human lymphocyte interferon gammaproduction by suppressing natural killer cell stimulatory factor/IL-12 synthesis in accessory cells. Journal of Experimental Medicine 178, 1041-1048.

Dai, W.J. \& Gottstein, B. (1999) Nitric oxide-mediated immunosuppression following murine Echinococcus multilocularis infection. Immunology 97, 107-116.

Dai, W.J., Hemphill, A., Waldvogel, A., Ingold, K., Deplazes, P., Mossmann, H. \& Gottstein, B. (2001) Major carbohydrate antigen of Echinococcus multilocularis induces an immunoglobulin $G$ response independent of alphabeta $+\mathrm{CD} 4+\mathrm{T}$ cells. Infection and Immunity 69, 6074-6083.

Dai, W.J., Waldvogel, A., Siles-Lucas, M. \& Gottstein, B. (2004) Echinococcus multilocularis proliferation in mice and respective parasite 14-3-3 gene expression is mainly controlled by an alphabeta CD4 T-cellmediated immune response. Immunology 112, 481-488.

Deplazes, P. \& Gottstein, B. (1991) A monoclonal antibody against Echinococcus multilocularis Em2 antigen. Parasitology 103, 41-49.

Dixon, J.B. (1997) Echinococcosis. Comparative Immunological and Microbiological Infectious Diseases 20, 87-94.

Dreweck, C.M., Soboslay, P.T., Schulz-Key, H., Gottstein, B. \& Kern, P. (1999) Cytokine and chemokine secretion by human peripheral blood cells in response to viable Echinococcus multilocularis metacestode vesicles. Parasite Immunology 21, 433-438.

Emery, I., Liance, M., Deriaud, E., Vuitton, D.A., Houin, R. \& Leclerc, C. (1996) Characterization of T-cell immune responses of Echinococcus multilocularisinfected C57BL/6J mice. Parasite Immunology 18, 463-472.

Godot, V., Harraga, S., Deschaseaux, M., Bresson-Hadni, S., Gottstein, B., Emilie, D. \& Vuitton, D.A. (1997) Increased basal production of interleukin-10 by peripheral blood mononuclear cells in human alveolar echinococcosis. European Cytokine Network 8, 401-408. 
Godot, V., Harraga, S., Podoprigora, G., Liance, M., Bardonnet, K. \& Vuitton, D.A. (2003) IFN alpha-2a protects mice against a helminth infection of the liver and modulates immune responses. Gastroenterology 124, 1441-1450.

Gottstein, B. \& Hemphill, A. (1997) Immunopathology of echinococcosis. Chemical Immunology 66, 177-208.

Gottstein, B., Deplazes, P. \& Aubert, M. (1992) Echinococcus multilocularis: immunological study on the 'Em2-positive' laminated layer during in vitro and in vivo post-oncospheral and larval development. Parasitology Research 78, 291-297.

Gottstein, B., Wunderlin, E. \& Tanner, I. (1994) Echinococcus multilocularis: parasite-specific humoral and cellular immune response subsets in mouse strains susceptible (AKR, C57B1/6J) or 'resistant' (C57B1/10) to secondary alveolar echinococcosis. Clinical Experimental Immunology 96, 245-252.

Harnett, W. \& Harnett, M.M. (2008) Therapeutic immunomodulators from nematode parasites. Expert Review of Molecular Medicine 10, e18.

Hemphill, A. \& Gottstein, B. (1995) Immunology and morphology studies on the proliferation of in vitro cultivated Echinococcus multilocularis metacestodes. Parasitology Research 81, 605-614.

Jenkins, S.J., Hewitson, J.P., Jenkins, G.R. \& Mountford, A.P. (2005) Modulation of the host's immune response by schistosome larvae. Parasite Immunology 27, 385-393.

Jenne, L., Kilwinski, J., Radloff, P., Flick, W. \& Kern, P. (1998) Clinical efficacy of and immunologic alterations caused by interferon gamma therapy for alveolar echinococcosis. Clinical Infectious Diseases 26, 492-494.

Jenne, L., Arrighi, J.F., Sauter, B. \& Kern, P. (2001) Dendritic cells pulsed with unfractionated helminthic proteins to generate antiparasitic cytotoxic T lymphocyte. Parasite Immunology 23, 195-201.

Konecny, P., Stagg, A.J., Jebbari, H., English, N., Davidson, R.N. \& Knight, S.C. (1999) Murine dendritic cells internalize Leishmania major promastigotes, produce IL-12 p40 and stimulate primary T cell proliferation in vitro. European Journal of Immunology 29, 1803-1811.

Liance, M., Bresson-Hadni, S., Meyer, J.P., Houin, R. \& Vuitton, D.A. (1990) Cellular immunity in experimental Echinococcus multilocularis infection. I. Sequential and comparative study of specific in vivo delayed-type hypersensitivity against E. multilocularis antigens in resistant and sensitive mice. Clinical and Experimental Immunology 82, 373-377.

Liance, M., Bresson-Hadni, S., Vuitton, D.A., Lenys, D., Carbillet, J.P. \& Houin, R. (1992) Effects of cyclosporin A on the course of murine alveolar echinococcosis and on specific cellular and humoral immune responses against Echinococcus multilocularis. International Journal of Parasitology 22, 23-28.

Liance, M., Ricard-Blum, S., Emery, I., Houin, R. \& Vuitton, D.A. (1998) Echinococcus multilocularis infection in mice: in vivo treatment with a low dose of IFN-gamma decreases metacestode growth and liver fibrogenesis. Parasite 5, 231-237.

Lightowlers, M.W. \& Rickard, M.D. (1988) Excretorysecretory products of helminth parasites: effects on host immune responses. Parasitology 96 (Suppl), S123-S166.

Lutz, M.B., Kukutsch, N., Ogilvie, A.L., Rössner, S., Koch, F., Romani, N. \& Schuler, G. (1999) An advanced culture method for generating large quantities of highly pure dendritic cells from mouse bone marrow. Journal of Immunological Methods 223, 77-92.

Macatonia, S.E., Hosken, N.A., Litton, M., Vieira, P., Hsieh, C.S., Culpepper, J.A., Wysocka, M., Trinchieri, G., Murphy, K.M. \& O'Garra, A. (1995) Dendritic cells produce IL-12 and direct the development of Th1 cells from naive CD4 + T cells. Journal of Immunology 154, 5071-5079.

MacDonald, A.S. \& Pearce, E.J. (2002) Cutting edge: polarized Th cell response induction by transferred antigen-pulsed dendritic cells is dependent on IL-4 or IL-12 production by recipient cells. Journal of Immunology 168, 3127-3130.

MacDonald, A.S., Straw, A.D., Bauman, B. \& Pearce, E.J. (2001) CD8- dendritic cell activation status plays an integral role in influencing Th2 response development. Journal of Immunology 167, 1982-1988.

MacDonald, A.S., Patton, E.A., La Flamme, A.C., Araujo, M.I., Huxtable, C.R., Bauman, B. \& Pearce, E.J. (2002) Impaired Th2 development and increased mortality during Schistosoma mansoni infection in the absence of CD40/CD154 interaction. Journal of Immunology 168, 4643-4649.

Maizels, R.M. \& Yazdanbakhsh, M. (2003) Immune regulation by helminth parasites: cellular and molecular mechanisms. Nature Review in Immunology 3, 733-744.

Margos, M. \& Gottstein, B. (2010) Gerbu adjuvant modulates the immune response and thus the course of infection in C56BL/6 mice immunised with Echinococcus multilocularis rec14-3-3 protein. Parasitology Research 107, 623-629.

Matsumoto, J., Müller, N., Hemphill, A., Oku, Y., Kamiya, M. \& Gottstein, B. (2006) 14-3-3- and II/3-10gene expression as molecular markers to address viability and growth activity of Echinococcus multilocularis metacestodes. Parasitology 132, 83-94.

Mejri, N. \& Gottstein, B. (2006) Intraperitoneal Echinococcus multilocularis infection in C57BL/6 mice affects CD40 and B7 costimulator expression on peritoneal macrophages and impairs peritoneal $\mathrm{T}$ cell activation. Parasite Immunology 28, 373-385.

Mejri, N., Hemphill, A. \& Gottstein, B. (2010) Triggering and modulation of the host-parasite interplay by Echinococcus multilocularis: a review. Parasitology 137, 557-568.

Mellman, I. \& Steinman, R.M. (2001) Dendritic cells: specialized and regulated antigen processing machines. Cell 106, 255-258.

Meyaard, L., Hovenkamp, E., Otto, S.A. \& Miedema, F. (1996) IL-12-induced IL-10 production by human T cells as a negative feedback for IL-12-induced immune responses. Journal of Immunology 156, 2776-2782.

Mosmann, T.R. (1994) Properties and functions of interleukin-10. Advances in Immunology 56, 1-26.

Müller, N., Frei, E., Nuñez, S. \& Gottstein, B. (2007) Improved serodiagnosis of alveolar echinococcosis of humans using an in vitro-produced Echinococcus multilocularis antigen. Parasitology 134, 879-888. 
Pasare, C. \& Medzhitov, R. (2004) Toll-dependent control mechanisms of CD4 T cell activation. Immunity 21, 733-741.

Perona-Wright, G., Jenkins, S.J. \& MacDonald, A.S. (2006) Dendritic cell activation and function in response to Schistosoma mansoni. International Journal of Parasitology 36, 711-721.

Playford, M.C., Ooi, H.K., Oku, Y. \& Kamiya, M. (1992) Secondary Echinococcus multilocularis infection in severe combined immunodeficient (scid) mice: biphasic growth of the larval cyst mass. International Journal of Parasitology 22, 975-982.

Rau, M.E. \& Tanner, C.E. (1975) BCG suppresses growth and metastasis of hydatid infections. Nature 256, 318-319.

Riganò, R., Buttari, B., Profumo, E., Ortona, E., Delunardo, F., Margutti, P., Mattei, V., Teggi, A., Sorice, M. \& Siracusano, A. (2007) Echinococcus granulosus antigen B impairs human dendritic cell differentiation and polarizes immature dendritic cell maturation towards a Th2 cell response. Infection and Immunity 75, 1667-1678.

Sakaguchi, S., Yamaguchi, T., Nomura, T. \& Ono, M. (2008) Regulatory $\mathrm{T}$ cells and immune tolerance. Cell 133, 775-787.

Sallusto, F. \& Lanzavecchia, A. (2002) The instructive role of dendritic cells on T-cell responses. Arthritis Research 4 (Suppl 3), S127-S132.

Siles-Lucas, M. \& Gottstein, B. (2003) The 14-3-3 protein: a key molecule in parasites as in other organisms. Trends in Parasitology 19, 575-581.

Siles-Lucas, M., Felleisen, R.S., Hemphill, A., Wilson, W. \& Gottstein, B. (1998) Stage-specific expression of the 14-3-3 gene in Echinococcus multilocularis. Molecular and Biochemical Parasitology 91, 281-293.

Siles-Lucas, M., Merli, M., Mackenstedt, U. \& Gottstein, B. (2003) The Echinococcus multilocularis 14-3-3 protein protects mice against primary but not secondary alveolar echinococcosis. Vaccine 21, 431-439.

Steinman, R.M. (2003) Some interfaces of dendritic cell biology. Apmis 111, 675-697.

Steinman, R.M. \& Nussenzweig, M.C. (2002) Avoiding horror autotoxicus: the importance of dendritic cells in peripheral T cell tolerance. Proceedings of the National Academy of Sciences USA 99, 351-358.

Stettler, M., Fink, R., Walker, M., Gottstein, B., Geary, T.G., Rossignol, J.F. \& Hemphill, A. (2003) In vitro parasiticidal effect of Nitazoxanide against Echinococcus multilocularis metacestodes. Antimicrobial Agents and Chemotherapy 47, 467-474.

Trinchieri, G. (1993) Interleukin-12 and its role in the generation of TH1 cells. Immunology Today 14, 335-338.

Trinchieri, G. (2003) Interleukin-12 and the regulation of innate resistance and adaptive immunity. Nature Review on Immunology 3, 133-146.

Varol, C., Landsman, L., Fogg, D.K., Greenshtein, L., Gildor, B., Margalit, R., Kalchenko, V., Geissmann, F. \& Jung, S. (2007) Monocytes give rise to mucosal, but not splenic, conventional dendritic cells. Journal of Experimental Medicine 204, 171-180.

Vuitton, D.A. (2003) The ambiguous role of immunity in echinococcosis: protection of the host or of the parasite? Acta Tropica 85, 119-132.

Walker, M., Baz, A., Dematteis, S., Stettler, M., Gottstein, B., Schaller, J. \& Hemphill, A. (2004) Isolation and characterization of a secretory component of Echinococcus multilocularis metacestodes potentially involved in modulating the host-parasite interface. Infection and Immunity 72, 527-536.

Wei, X.L., Ding, J.B., Xu, Y., Wen, H. \& Lin, R.Y. (2004) Change of cytokines in mice with Echinococcus multilocularis infection. Zhongguo Ji Sheng Chong Xue Yu Ji Sheng Chong Bing Za Zhi 22, 361-364 (in Chinese). 